\title{
Una vez más sobre el texto de Filénide, Frag. 3, Col. II (P. Oxy. XXXIX 2891)
}

\author{
Miguel Ángel Spinassi ${ }^{1}$
}

Recibido: 20 de Abril de 2019 / Aceptado: 28 de Mayo de 2019

Resumen. Desde que E. Lobel publicó en 1972 tres fragmentos de un papiro que contienen parte del escrito amatorio de Filénide de Samos muchas conjeturas se propusieron para resolver las principales dificultades de lectura. En esta ocasión intento una reconstrucción de la segunda columna del tercer fragmento conservado a fin de arrojar luz sobre los principales puntos oscuros del texto que, se supone, contiene indicaciones sobre cómo adular a la mujer amada.

Palabras clave: Filénide; seducción; papiro; reconstrucción.

\section{[en] Once again on the Text of Philaenis, Fr. 3, Col. II (P. Oxy. XXXIX 2891)}

\begin{abstract}
Since E. Lobel published in 1972 three fragments of a papyrus that contain part of the writings of Philaenis from Samos, many conjectures were proposed to solve the main difficulties of reading. On this occasion, I intend a reconstruction of the second column of the third fragment preserved in order to shed light on the main dark points in the text that is supposed to contain indications of how to flatter the beloved woman.
\end{abstract}

Keywords: Philaenis; seduction; papyrus; reconstruction.

Cómo citar: Spinassi, M. A. (2020) Una vez más sobre el texto de Filénide, Frag. 3, Col. II (P. Oxy. XXXIX 2891), en Cuadernos de Filología Clásica. Estudios griegos e indoeuropeos 30, 125-130.

1 Universidad Nacional de Córdoba (Argentina), Escuela de Letras, FFyH

miguel.spinassi@unc.edu.ar 
De Filénide, oriunda de la isla de Samos, se han conservado tres fragmentos ${ }^{2}$. En esta ocasión quisiera releer el tercer fragmento que, se supone, sería la parte conclusiva del apartado "sobre la seducción" ( $\pi \varepsilon \rho \grave{i} \pi \varepsilon i \rho \alpha c \mu \tilde{v})$ del fr. 2. A continuación cito el texto de la edición de E. Lobel (1972) y adjunto un aparato crítico con las variantes de lectura más importantes que se han propuesto hasta el momento:

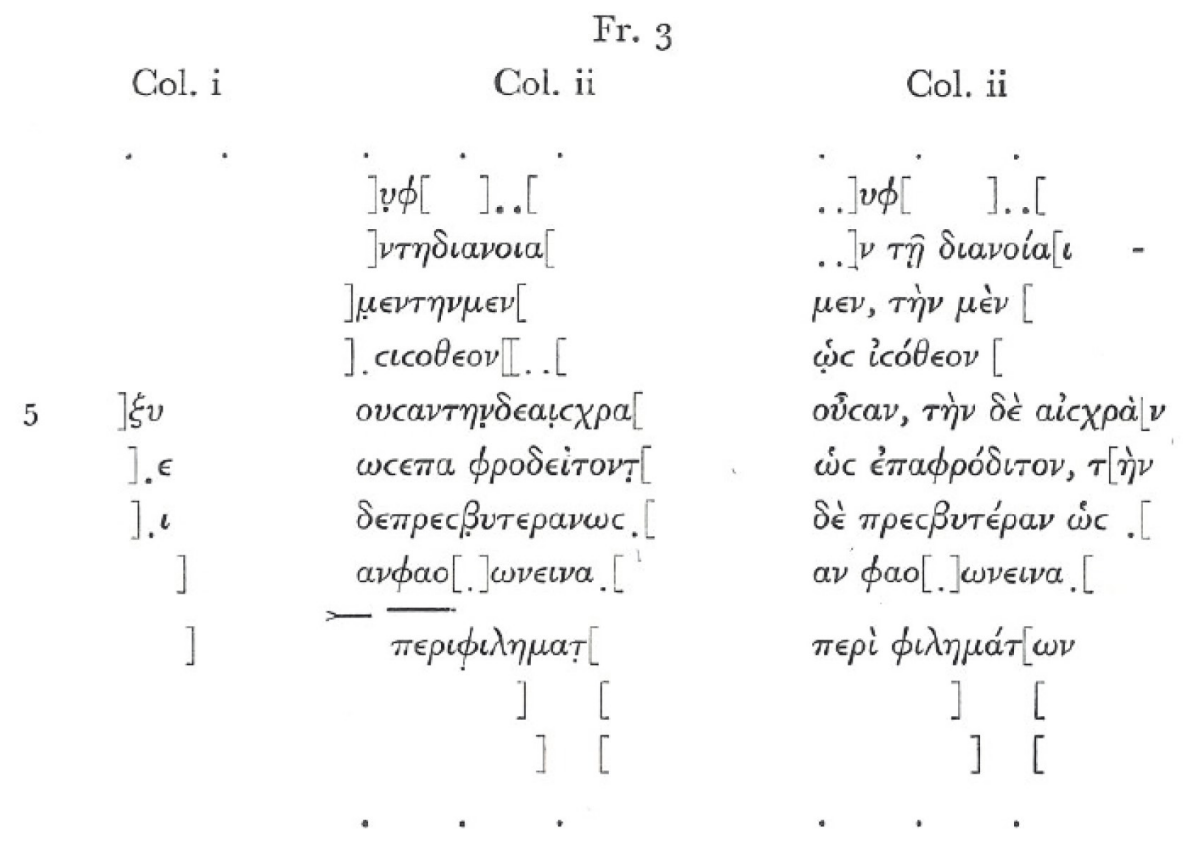

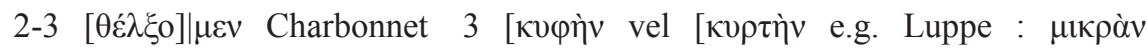

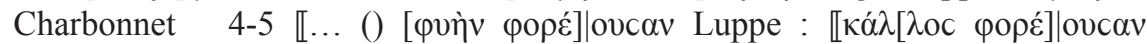

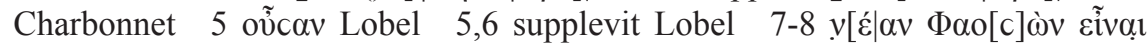

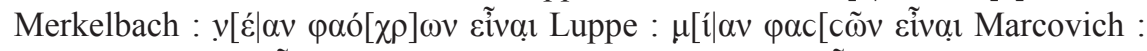

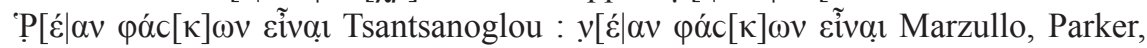
Whitehorne : $\theta[\varepsilon] \mid \grave{\alpha} v$ Charbonnet, Perale $8 \Phi \alpha o[v] \omega v \varepsilon i ́ v \alpha v$ Lobel

Como advirtió Lobel, el fragmento parece contener el mismo motivo que Pl. $R$. 474d ss., Lucr. 4.1153-1170, y Ov. Ars. 2.657-662 (cf. Rem. 327-330). En líneas generales, y de manera más o menos explícita, estos tres pasajes se refieren a cómo las cualidades negativas de la persona amada son suavizadas o alabadas por el

2 Quisiera agradecer a M. Perale (Universidad de Liverpool) por facilitarme el texto en formato pdf. del archivo de Charbonnet, cuyo link (citado en Perale 2013: 127, n. 2) actualmente no funciona; al profesor J. Hammerstaedt (Universidad de Colonia) por sus valiosos comentarios sobre una versión previa de este trabajo y a los evaluadores de la revista $C F C(G)$ por sus atinadas observaciones.

Para una discusión al respecto de la existencia de Filénide y su obra, véase Whitehorne (1990) y Boehringer (2015). Una revisión de los pasajes de la literatura antigua que aluden a esta Filénide se puede leer en Herrero Ingelmo \& Montero Cartelle (1990) y Agnolon (2013). En lo que sigue todas las traducciones al castellano son mías. 
amante con una expresión eufemística. En esta dirección deberían ir los esfuerzos de reconstrucción del fragmento, puesto que parece claro que Filénide también intenta aconsejar al lector sobre cómo proceder, en este caso, con los defectos de la mujer amada ${ }^{3}$.

En nuestra porción de papiro conservada como fr. 3 hay dos marcas textuales

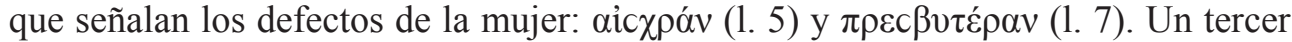
defecto sería esperable hacia el final de la tercera línea. Por otra parte, no queda claro cuál sería la virtud contrapuesta a este tercer defecto (final de línea 4) que sería calificada de "igual a un dios" (icó $\theta \varepsilon o v$ ), siendo que las otras dos calificaciones positivas sí pueden ser reconstruidas con un aceptable consenso entre los especialistas

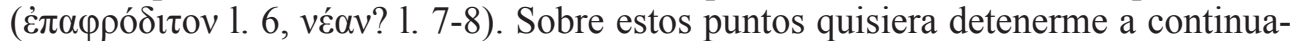
ción y hacer alguna conjetura plausible.

Antes de ello, una consideración sobre la línea 2-3. La lectura del dativo $\tau \tilde{n}$ $\delta$ ¿avoí $\alpha[1$, posiblemente precedido de la preposición $\varepsilon \dot{\varepsilon}[v$, y la terminación $-\mu \varepsilon v$ del comienzo de la tercera línea, quizás la primera persona del plural, nos hace su-

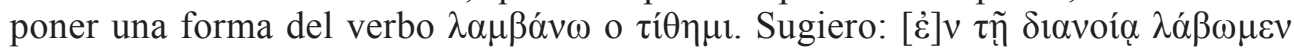
" $<$ lo $>$ tengamos en la mente", " $<$ lo $>$ imaginemos", etc. Filénide parece incluirse así en el acto de seducción e invita a continuación a "tener presente" cómo se ha de llamar a la mujer amada que tiene cualidades negativas. Esta reconstrucción tendría la ventaja, si tiene alguna, de ofrecer el verbo principal de la oración que regirá la subordinada de infinitivo, como se desprende hacia la parte conclusiva de la columna (1. 8).

Los finales de la tercera y cuarta línea serán siempre una incógnita. Para una conjetura verosímil hay que atender a la siguiente porción del texto que tiene un mejor estado de preservación. Si el final de 1. 7 debe ser completado con [ve- para formar

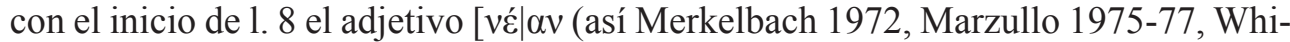
tehorne 1990, y Luppe 1998), está bastante claro que la intención de la autora ha sido

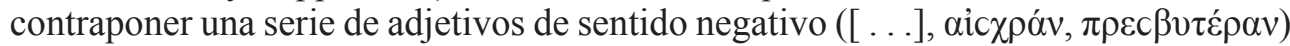

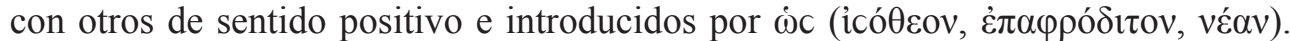
Ahora bien, ¿cuál podría ser el final de esa tercera línea que incluiría un calificativo

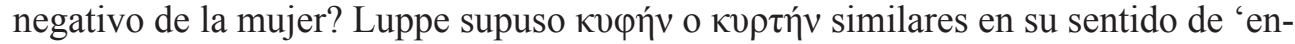

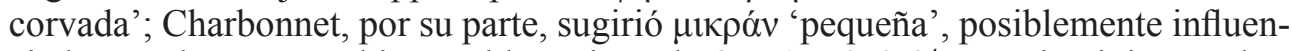
ciado por el texto -también problemático- de Ov. Ars. 2.6594. En mi opinión, ambas propuestas irían por el camino correcto, puesto que el adjetivo esperado tiene que ver con el aspecto físico de la mujer. En esta dirección, sería quizás oportuno reparar en un pasaje de Juan Crisóstomo, patriarca de Constantinopla, en el que encontramos una secuencia de adjetivos similar a la que podríamos esperar en el texto de Filénide y que arrojaría luz a lo que falta por completar. Se trata casualmente de un escrito sobre el matrimonio en el que se compara la relación entre Cristo-Iglesia con la que un

3 En la primera línea solo se lee ] $v \varphi[$ y los trazos de dos o tres letras más hacia el final. No es posible aven-

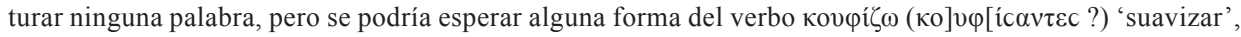
'ablandar', solo por influencia de Ov. Ars. 2.657 "nominibus mollire licet mala” etc., o también una del

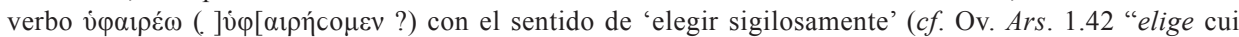
dicas 'tu sola mihi places"”), o incluso 'robar', quizás metafóricamente en un contexto de adulterio ( $c f$. D. Chr. 74.20.10; Lib. Decl. 38.1.45.2). Para una consideración sobre Filénide como fuente del Ars amatoria de Ovidio, véase Cataudella (1974) y Thomson Vessey (1976: 82). Incluso algunos, como Alfonsi (1974), han extendido la influencia de la escritora griega hasta Propercio y Marcial.

$4 \quad$ El manuscrito parisino $R$ (apud Bornecque 1960) lee parua, que Heinsius corrigió en raua. Cf. Lucr. 4.1162 parvula, pumilio. 
hombre debería tener con su esposa en el momento de contraer matrimonio (Chrys. laud. Max. 51.228.17-21):

Y esto no solo es asombroso, a saber, después de tomar una [sc. mujer] deforme, de fea figura, despreciable y vieja (sc. la Iglesia), no sintió repugnancia por su fealdad, sino que se entregó a la muerte y [al hacerlo] la transformó en una belleza increíble.

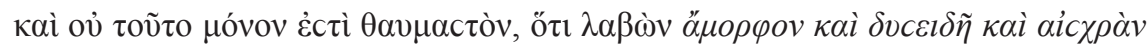

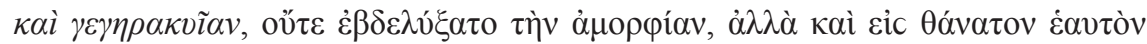

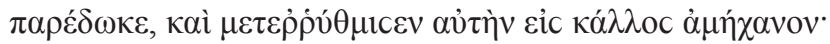

Aun cuando este pasaje sea tardío, es probable que Juan conociera el texto de Filénide u otros de carácter amatorio contra los cuales reaccionaría en sus propios escritos, como este del Encomio a Máximo, que justamente lleva el subtítulo de Quales sint ducendae uxores, tal como lo hicieron los primeros padres apologéticos cristianos ( $c f$. Clem. Al., Protr. 4.61.2-3). Parece verosímil, pues, que la tercera línea de nuestro papiro haya contenido un adjetivo del tipo ő $\mu \rho \rho \varphi v$ o $\delta v c \varepsilon i \delta \tilde{\eta}$, que, por otra parte, solo excedería en una letra a la palabra más larga propuesta por Luppe, quien consideraba que el papiro solo admite espacio "para un breve adjetivo" (1998: 87). Respecto de las propuestas anteriores, ő $\mu$ o $\varphi$ ov o $\delta v c \varepsilon 1 \delta \tilde{\eta}$ tienen la ventaja de no ser tan arriesgadas en tanto aluden al aspecto físico general de la mujer y no a un defecto particular (cf. D.L. 4.4.4).

El final de la cuarta línea parece contener el trazo de un par de letras que han sido canceladas y marcadas por encima con un punto, quizás la sílaba $\omega c$, como anota Lobel. Si ello es así, estaríamos ante la repetición errónea del adverbio ஸ́c, que acompaña a cada adjetivo positivo, que alguna mano eliminó al reconocer la equivocación. Lobel, en última instancia, cree que "nada debería estar perdido" allí y por ello lee el comienzo de la quinta línea como el participio presente femenino oũcav. Contra esto, está la posición de Tsantsanoglou (1973: 189), quien entiende que -ovcav es el final de un participio iniciado en la línea precedente y terminado en una vocal. Por

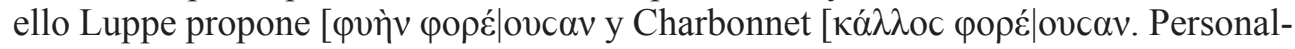
mente creo que una expresión como la sugerida por Charbonnet podría tener sentido aquí. En muchos contextos el adjetivo icó $\theta \varepsilon o c$ está referido al ser humano ( $\varphi \omega ́ c$, en Homero, passim), a la estima u honra, y a veces a la belleza, cuyo ejemplo más im-

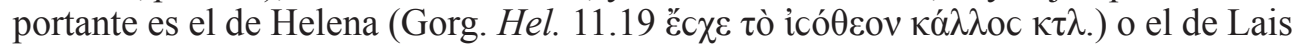

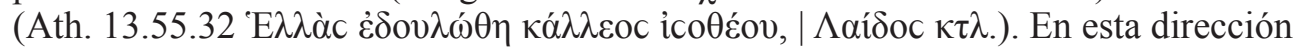

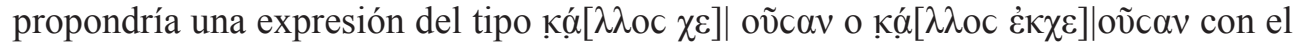
sentido de "... vertiendo belleza", metáfora que en algunas zonas de habla hispana perdura en la expresión "derrochar belleza", y que podría ser una de las formas

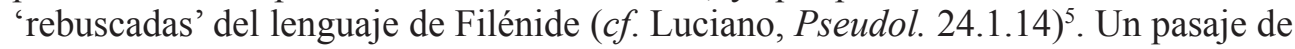
los Moralia de Plutarco podría apoyar esta lectura, allí donde se habla del mal de ojo y el poder que tiene la mirada de las personas: los placeres amorosos, se dice, se

También estuve vacilante a la hora de aceptar otra propuesta en esta porción del texto: el segundo de los dos trazos de las letras supuestamente canceladas se asemeja mucho a una $\delta$. Influenciado por el pasaje de Juan

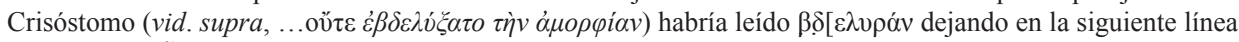
el participio oũcav como ya había propuesto Lobel. 
inician con la vista, de manera que lo ‘erótico' fluye y se funde cuando el alma mira las personas bellas, "como si se derramara sobre ellas" (Plu. Moralia 681 B3 ...oĩov

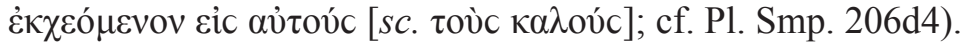

La última línea plantea un problema importante. Tsantsanoglou (1973) sugiere que el trazo casi cerrado de la ómicron en realidad pertenece a una sigma y lee el participio presente $\varphi a ́ c[\kappa] \omega v$, que concordaría con el sujeto ó $\pi \varepsilon 1 \rho \tilde{\omega} v$, el seductor, de la columna ii del fragmento 1. La expresión $\varphi \alpha ́ c \kappa \omega v$ eĩvol no repugnaría y sería un cierre perfecto para el texto. No estoy seguro, sin embargo, de que el trazo sea efectivamente una sigma ni de la explicación de Tsantsanoglou. Luppe (1974) propuso la atractiva lectura de $\varphi \alpha o ́[\chi \rho] \omega v$, 'de piel clara como la luz', adjetivo que, si bien no está atestiguado, sería digno de atención. Quizás el final del pasaje platónico de la República aludido más arriba pueda apoyar la intuición de Luppe, allí donde se dice que los amantes llaman a quienes son 'blancos' o quizás 'pálidos' como "hijos

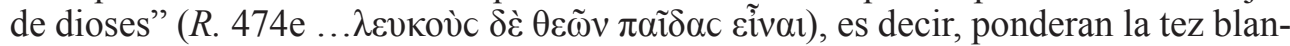
ca como un rasgo divino. En este sentido la piel blanca y tersa de la mujer "como la luz" se contrapondría así a las arrugas y quizás a las manchas de la vejez. En la Anthologia Palatina, por otra parte, encontramos algunas referencias al buen estado de conservación de las frutas que los amantes ofrecen a sus enamoradas como símbolo del deseo. La nota más distintiva de estas frutas es la conservación de su piel en un estado siempre "nuevo", algo que quizás habría estado presente también en la mente de Filénide. Así, tenemos que en AP 6.252 se presenta un membrillo conser-

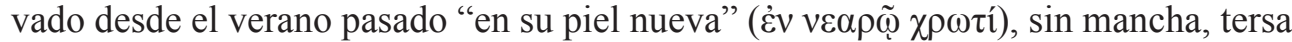
y sin arrugas $^{6}$. El ejemplo más significativo, sin embargo, podría ser el Epigrama 9 de Filodemo (ed. Sider), en donde se alaban los atributos físicos de una mujer que, a pesar de sus años, conserva su piel sin arrugas y sus pechos marmóreos, i.e. "blancos", todavía firmes.

Por todo esto, podría conjeturar así el texto de nuestro fragmento:

$$
\begin{aligned}
& . .] v \varphi[\quad] \ldots[\quad]
\end{aligned}
$$

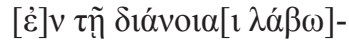

$$
\begin{aligned}
& \mu \varepsilon v \tau \eta ̀ v ~ \mu \varepsilon ̀ v ~[\alpha ̋ \mu \rho \rho \varphi \nu]
\end{aligned}
$$

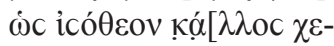

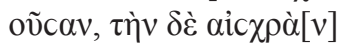

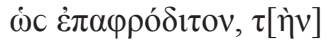

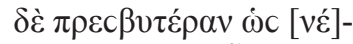

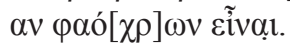

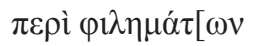

$<_{i}$...habiendo suavizado sus defectos?>, consideremos en nuestro pensamiento que aquella que es contrahecha derrama una belleza divina, que la repulsiva es deseable al igual que Afrodita, y que la que ya es mayor es una joven de piel tan blanca como la luz.

Sobre los besos

6 Vid., además, AP 6.102, y Hor. Carm. 1.19.6 "urit me Glycerae | nitor splendentis Pario marmore purius". 


\section{Bibliografía}

Agnolon, Alexandre (2013), «Filênis, de belle de jour à alcoviteira: matéria erótica na Antologia Grega», Revista Brasileira de Estudos Clássicos 26.1: 51-66.

Alfonsi, Luigi (1974), «Da Filenide a Properzio», Aegyptus 54. 1/4: 176-178.

Boehringer, Sandra (2015), «What is named by the name of Philaenis?», en M. Masterson, N. S. Rabinowitz \& J. Robson (eds.), Sex in Antiquity. Exploring Gender and Sexuality in the Ancient World, London-New York, Routledge: 374-392.

Bornecque, Henri (1960), Ovide. L'art d'aimer, Paris, Belles Lettres.

Cataudella, Quintino (1974), «Initiamenta Amoris», Latomus 33.4: 847-857.

Herrero Ingelmo, María Cruz \& Montero Cartelle, Enrique (1990), «Filénide en la literatura grecolatina», Euphrosyne 18: 265-274.

Luppe, Wolfgang (1974), «Nochmals zu Philainis, Pap.Oxy. 2891», ZPE 13: 281-282. Luppe, Wolfgang (1998) «Zum Philainis-Papyrus (P. Oxy. 2891)», ZPE 123: 87-88.

Marzullo, Benedetto (1975-77), «Philaenis P. Oxy. 2891, fr. 3», MCr 10-12: 173-175.

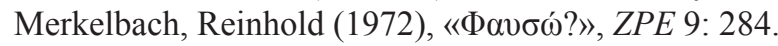

Parker, Holt N. (1989), «Another Go at the Text of Philaenis (P. Oxy. 2891)», ZPE 79: 49-50. Perale, Marco (2013), «Philainis und Aristoteles? Zum Philainis-Papyrus P. Oxy. 2891», en P. Mauritsch (ed.), Aspekte antiker Prostitution, Graz, Grazer Universitätsverlag: 127-135.

Thomson Vessey, D. W (1976), «Philaenis», Revue Belge de Philologie et d'Histoire 54.1: 78-83.

Tsantsanoglou, Kyriakos (1973), «The Memoirs of a Lady from Samos», ZPE 12: 183-195.

Whitehorne, John Edwin George (1990), «Filthy Philaenis (P. Oxy. XXXIX 2891): A real Lady?», en M. Capasso, G. Messeri Savorelli \& R. Pintaudi (eds.), Miscellanea Papyrologica in occasione del bicentenario dell'edizione della Charta Borgiana, Firenze, Edizioni Gonnelli: 529-542. 